\title{
CALIBRATION OF CYCLIC FORCE WITH INERTIAL FORCE CORRECTION TO A FATIGUE TESTING MACHINE
}

\author{
Tian Feng ${ }^{1}$, Yin $\mathrm{Xiao}^{1}, \mathrm{Li} \mathrm{Bo}^{1}$ \\ ${ }^{1}$ Changcheng Institute of Metrology and Measurement, Beijing, China, tfpu@sina.com
}

\begin{abstract}
:
The accuracy between a dynamic force and a static force applied on a specimen by a fatigue machine is usually not the same. By establishing physical vibration models of fatigue machines, it is concluded that the error of a cyclic force is mainly caused by the inertial force of the vibration mass between the machine sensor and the specimen. After the inertial force is exactly corrected, the force displayed on the machine would be consistent with the real force on the specimen. A standard dynamic force calibration sensor (DFCS) with an inertial force correction method has been used to do calibration of fatigue testing machines in this paper. Compared with the replica test-piece method, the two calibration results are close to each other.
\end{abstract}

Keywords: fatigue machine; calibration; dynamic force; inertial force; correction

\section{INTRODUCTION}

The purpose of cyclic force calibration is to make the indication force of a machine sensor consistent with the actual force loaded on the specimen. Replica test-piece method in ISO 4965-1 and ASTM E467-08 has been widely used in fatigue machine calibration $[1,2]$. In this method, there are some strict limitations of a calibration device regarding to stiffness and mass. Because standard force sensors are widely used in various fields, they are expected to be used to calibrate fatigue machines, even though they usually don't have the same stiffness and mass with a real specimen. By analysing vibration models of some fatigue machines, it is concluded that some types of machines do not need to have the same stiffness of a DFCS as the real specimen. It also suggests that although use a calibration device giving different mass and stiffness from that of a real specimen, doing inertial force correction would lead to the same result to the replica test-piece. The difference between the results given by the two methods is within $1 \%$.

\section{ANALYSIS OF THE INERTIAL FORCE CORRECTION MODEL}

The dynamic error of a cyclic force generally comes from two aspects: the performance of the machine force measuring system and the influence of the inertial force. The cyclic force error caused by the performance of a machine force measuring system could be avoided or eliminated by selecting a force measuring system with a better dynamic performance. The error caused by an inertial force cannot be avoided and eliminated, it could only be induced as little as possible or corrected as accurately as possible. The difference between a machine sensor force and a specimen real force is due to the fact that the machine sensor and the specimen are not in the same position. There is a component mass that cannot be ignored between them, and the connection stiffness between them is limited rather than infinite. During a dynamic test, the mass of the component between them has an acceleration, so that the inertial force is generated. For the reason that the inertial force does not act on the sensor and the specimen symmetrically, an error of the cyclic force is produced. If the exact value of the inertial force influence is known, the actual force loaded on a specimen could be derived, or calibration to a testing machine force could be done. An analysis of the vibration system model of a fatigue testing machine is needed to determine the influence of an inertial force.

Local modelling concerned with the force calibration is carried out. The local vibration segment contains all the parts between the testing machine sensor and the specimen. Only the mass and stiffness within this segment are considered, while those beyond this segment are ignored. In this case, any fatigue testing machine could be analysed simply as a two-degree-of-freedom vibration model, as shown in Figure 1 [3]. 


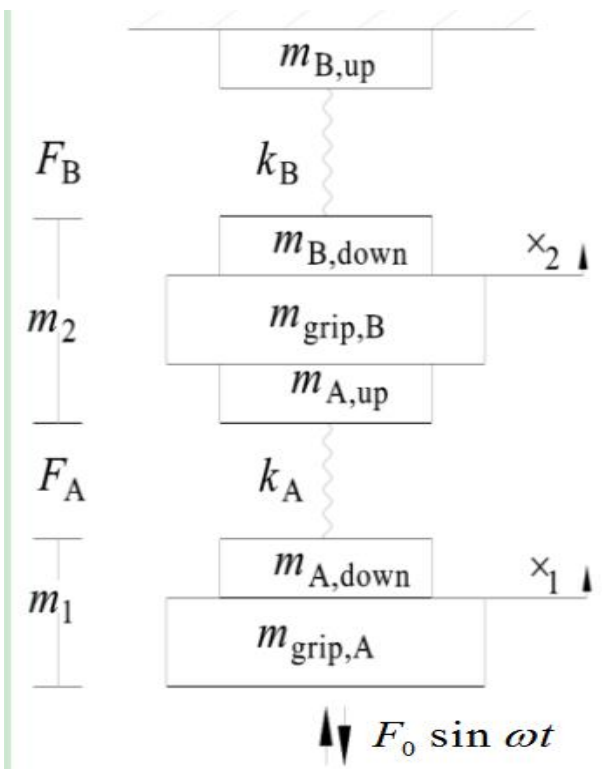

Figure 1: Local vibration model of fatigue testing machines

According to D'Alembert's principle, the two differential equations of the vibration system are:

$m_{1} \ddot{x}_{1}+k_{\mathrm{A}}\left(x_{1}-x_{2}\right)=F_{0} \sin \omega t$

$m_{2} \ddot{x}_{2}+k_{\mathrm{B}} x_{2}-k_{\mathrm{A}}\left(x_{1}-x_{2}\right)=0$

The inertial force $F_{\mathrm{i}}$ is as follows:

$$
F_{\mathrm{i}}=k_{\mathrm{B}} \Delta x_{2}-k_{\mathrm{A}} \Delta x_{1}=m_{2} \Delta x_{2} \omega^{2}
$$

The relative inertial force $F_{\text {rel,i }}$ is as follows:

$$
F_{\text {rel, }, \mathrm{i}}=\frac{F_{\mathrm{i}}}{F_{\mathrm{B}}}=\frac{m_{2} \Delta x_{2} \omega^{2}}{k_{\mathrm{B}} \Delta x_{2}}=\frac{m_{2} \omega^{2}}{k_{\mathrm{B}}}
$$

where:

$m_{1}, m_{2}$ - the equivalent mass at positions $\mathrm{A}$ and $\mathrm{B}$, in $\mathrm{kg}$;

$k_{\mathrm{A}}, k_{\mathrm{B}}$ - the stiffness at positions $\mathrm{A}$ and $\mathrm{B}$, in $\mathrm{N} \cdot \mathrm{m}^{-1}$

$x_{1}, x_{2}$ - the instantaneous displacements of the elastic elements at positions $\mathrm{A}$ and $\mathrm{B}$, in $\mathrm{mm}$;

$\Delta x_{1}, \Delta x_{2}$ - the instantaneous deformation of the elastic elements at positions $\mathrm{A}$ and $\mathrm{B}$, in $\mathrm{mm}$;

$\omega$ - the angular frequency of a fatigue testing machine, in $\mathrm{rad} \cdot \mathrm{s}^{-1}$;

$F_{\mathrm{A}}, F_{\mathrm{B}}$ - the real force between positions $\mathrm{A}$ and $\mathrm{B}$, in $\mathrm{kN}$;

$F_{\mathrm{i}}$ - the inertial force between positions $\mathrm{A}$ and B, in $\mathrm{kN}$.

Equation (3) above indicates that a relative inertial force is associated with $m_{2}$ and $k_{\mathrm{B}}$. Figure 1 illustrates two positions, position A and B. Position $\mathrm{A}$ is far from the motionless end, on the contrary, position $\mathrm{B}$ is close to the motionless end. Correspondingly, there are usually two configurations of fatigue machines. One is that the machine sensor lies at position $\mathrm{A}$ and the test specimen lies at position B - Type 1 configuration. The other one is exactly the opposite, that is the test specimen lies at position $\mathrm{A}$ and the machine sensor lies at position B - Type 2 configuration.

For Type 1 configuration that $\mathrm{A}$ is the machine sensor and B is the test specimen, both the stiffness and mass of a specimen are the influence factors of an inertial force. The mass and stiffness of every different specimen have to be measured, and the correction of the inertial force induced by DFCS, which has different mass and stiffness from the real specimen, has to be done. The function between the real force loaded on the specimen and the machine sensor force is expressed by equation (4), whereas the function between the DFCS force and the machine sensor force is expressed by equation (5).

$F_{\mathrm{A} 1}=F_{\mathrm{B} 1}\left(1-\frac{m_{2} \omega^{2}}{k_{\mathrm{B}}}\right)$

$F_{\mathrm{A} 2}=F_{\mathrm{B} 2}\left(1-\frac{m_{2}^{\prime} \omega^{\prime 2}}{k_{\mathrm{B}}^{\prime}}\right)$

$=F_{\mathrm{B} 2}\left[1-\frac{\left(m_{2}+\Delta m\right)(\omega+\Delta \omega)^{2}}{k_{\mathrm{B}}+\Delta k}\right]$

$=F_{\mathrm{B} 2}\left[1-\frac{m_{2} \omega^{2}}{k_{\mathrm{B}}}-\frac{m_{2}\left(2 \omega \Delta \omega+\Delta \omega^{2}\right)}{k_{\mathrm{B}}}\right.$

$\left.-\frac{\left(\Delta m k_{\mathrm{B}}-m_{2} \Delta k\right)(\omega+\Delta \omega)^{2}}{k_{\mathrm{B}}\left(k_{\mathrm{B}}+\Delta k\right)}\right]$

In equation (5), $\Delta m$ is the increment of $m_{2}$ after the real specimen is replaced by a DFCS in calibration. Similarly, $\Delta k$ is the increment of $k_{\mathrm{B}}$, and $\Delta \omega$ is the increment of $\omega$. Where for nonresonant fatigue machine, $\Delta \omega=0$.

From equation (4), the dynamic error of a machine force obtained by a replica test piece is as follows:

$\delta=\frac{F_{\mathrm{A} 1}-F_{\mathrm{B} 1}}{F_{\mathrm{B} 1}}=-\frac{m_{2} \omega^{2}}{k_{\mathrm{B}}}$

From equation (5), the dynamic error of a machine force obtained by a DFCS is as follows. Note that equation (7) is equivalent to equation (6).

$\delta=\frac{1}{F_{\mathrm{B} 2}}\left[\begin{array}{l}F_{\mathrm{A} 2}-F_{\mathrm{B} 2}+F_{\mathrm{B} 2} \frac{m_{2}\left(2 \omega \Delta \omega+\Delta \omega^{2}\right)}{k_{\mathrm{B}}} \\ +F_{\mathrm{B} 2} \frac{\left(\Delta m k_{\mathrm{B}}-m_{2} \Delta k\right)(\omega+\Delta \omega)^{2}}{k_{\mathrm{B}}\left(k_{\mathrm{B}}+\Delta k\right)}\end{array}\right]$

For Type 2 configuration that $\mathrm{A}$ is a test specimen and $\mathrm{B}$ is a machine sensor, $F_{\mathrm{A}}$ is the real force of the specimen, and $F_{\mathrm{B}}$ is the machine sensor force. Because the specimen stiffness is $k_{\mathrm{A}}$ instead of $k_{\mathrm{B}}$ in this case, it is no longer the influence factor of the inertial force, given in equation (3). Therefore, for this type of machine, the DFCS does not have to have the same stiffness as the real specimen. However, the correction of the inertial force could still be done using equation (7), where $\Delta k=0$. 
The inertial force $F_{\mathrm{i}}$ could also be expressed as a function of the acceleration as follows:

$F_{\mathrm{i}}=k_{\mathrm{B}} \Delta x_{2}-k_{\mathrm{A}} \Delta x_{1}=m_{2} \Delta x_{2} \omega^{2}=m a$

Equations (4) and (5) can be expressed equivalently as equation (10) and (11). Where in equation (11), $\Delta a$ is the acceleration increment of $m_{2}$ after the real specimen is replaced by a DFCS.

$F_{\mathrm{A} 1}=F_{\mathrm{B} 1}-m_{2} a$

$F_{\mathrm{A} 2}=F_{\mathrm{B} 2}-m_{2}^{\prime} a^{\prime}$

$=F_{\mathrm{B} 2}-\left(m_{2}+\Delta m\right)(a+\Delta a)$

$=F_{\mathrm{B} 2}-m_{2} a-\Delta m(a+\Delta a)-m_{2} \Delta a$

Equations (6) and (7) can be expressed equivalently as equation (12) and (13).

$\delta=\frac{F_{\mathrm{A} 1}-F_{\mathrm{B} 1}}{F_{\mathrm{B} 1}}=-\frac{m_{2} a}{F_{\mathrm{B} 1}}$

$\delta=\frac{1}{F_{\mathrm{B} 1}}\left[F_{\mathrm{A} 2}+\Delta m(a+\Delta a)+m_{2} \Delta a-F_{\mathrm{B} 2}\right]$

For Type 2 configuration of non-resonant fatigue machine, when $F_{\mathrm{B} 1}=F_{\mathrm{B} 2}$ in equation (13), $\Delta a=0$.

In a cyclic force calibration, the real specimen is replaced with a DFCS. Thus, the extra inertial force induced by the difference of the mass and stiffness of the DFCS from the real specimen should be corrected.

To do the correction to the cyclic force, the first step is to measure $m_{2}$ and $k_{\mathrm{B}}$, then the second step is to measure $\Delta m$ and $\Delta k$ for Type 1 configuration, or only $\Delta m$ for Type 2 configuration.

Similarly, for acceleration method correction, the first step is to measure $m_{2}$ and $a$, and the second step is to measure $\Delta m$ and $\Delta a$ for Type 1 configuration, or only $\Delta m$ for Type 2 configuration.

\section{EXPERIMENTS AND DISCUSSION}

\subsection{Composition of the Calibration System}

A cyclic force calibration system with the inertial force correction function consists of a dynamic force measurement subsystem, an inertial force correction subsystem, an alignment measurement subsystem, and a data acquisition and processing subsystem. The dynamic force measurement subsystem includes a DFCS subsystem and a replica test-piece subsystem. A set of DFCS consists of some standard dynamic force sensors with different force ranges, as shown in Figure 2. The inertial force correction subsystem includes an acceleration measurement subsystem and a stiffness/displacement measurement subsystem, as shown in Figure 3.

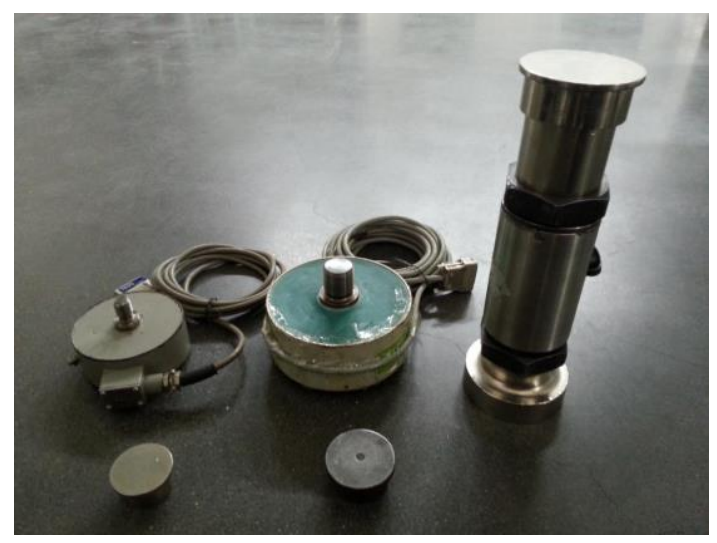

Figure 2: DFCS

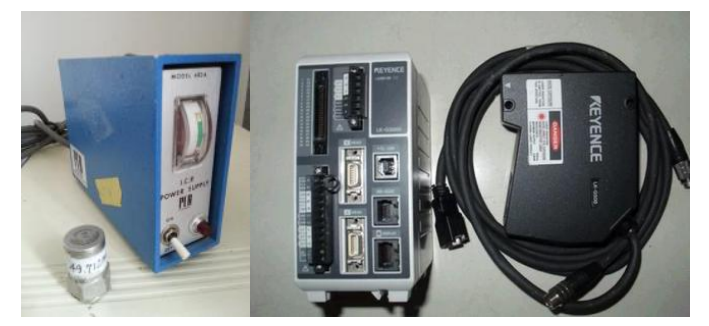

Figure 3:The inertial force correction subsystem

\subsection{Methodology and Procedure of Tests}

Tests were done in an electromagnetic resonance fatigue machine with controlled force, as shown in Figure 4. There is an inertial mass compensation function for this machine, but this function is not used in tests so that the inertial force compensation function of the DFCS could be verified.
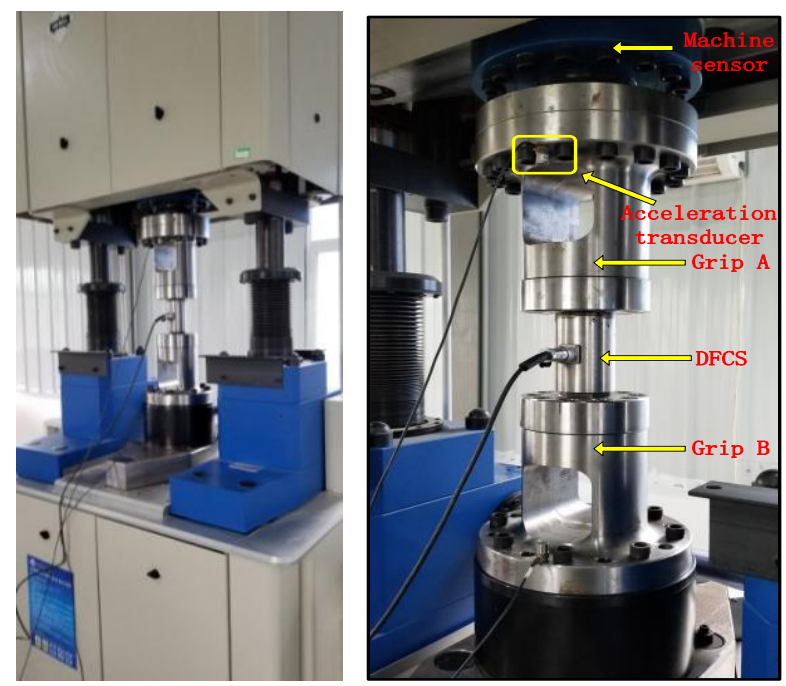

Figure 4: Calibration to an electromagnetic resonance fatigue testing machine with a DFCS

Firstly, use a replica test piece for calibration, and secondly, use a DFCS. When a DFCS is being used, a stiffness method or an acceleration method for additional inertial force correction to the DFCS are simultaneously used. The DFCS is connected to the fatigue testing machine with proper adapters, and the acceleration transducer is installed on the vibration mass between the DFCS and the testing machine sensor. Set up the dynamic test force of the fatigue testing machine and start the machine. When 
the dynamic test force reaches the target value and the sine wave is stable, collect the peak and valley values of 50 cycle waveforms, and record the test frequency and the acceleration value. The displacement data are collected at the same time with the former static force calibration, and then the stiffness value is calculated.

\subsection{Data of Tests}

The calibration data is shown in Table 1 and Table 2. The calculations for the relative error of the cyclic force are given in equations (6), (7), (12), and (13) and the calculation parameters are shown in Table 3. The sample rate is $5 \mathrm{kHz}$, Bessel filter frequency is $500 \mathrm{~Hz}$.

Table 1: Calibration data of the replica test piece

\begin{tabular}{|c|c|c|c|c|c|c|r|r|r|}
\hline $\begin{array}{c}\text { Data of } \\
\text { the } \\
\text { replica } \\
\text { test } \\
\text { piece }\end{array}$ & $\begin{array}{c}\text { Test } \\
\text { frequency }\end{array}$ & $\begin{array}{c}\text { Peak force } \\
\text { of the } \\
\text { machine } \\
/ \mathbf{~ k N}\end{array}$ & $\begin{array}{c}\text { Valley } \\
\text { force of } \\
\text { the } \\
\text { machine } \\
/ \mathbf{k N}\end{array}$ & $\begin{array}{c}\text { Amplitude } \\
\text { of the } \\
\text { machine } \\
\text { force } \\
/ \mathbf{k N}\end{array}$ & $\begin{array}{c}\text { Peak force } \\
\text { of the test } \\
\text { piece } \\
/ \mathbf{k N}\end{array}$ & $\begin{array}{c}\text { Valley } \\
\text { force of } \\
\text { the test } \\
\text { piece } \\
/ \mathbf{k N}\end{array}$ & $\begin{array}{c}\text { Amplitude } \\
\text { of the test } \\
\text { piece force } \\
/ \mathbf{k N}\end{array}$ & $\begin{array}{c}\text { Error of } \\
\text { the } \\
\text { machine } \\
\text { amplitude } \\
/ \%\end{array}$ & $\begin{array}{c}\text { Difference } \\
\text { from } \\
\text { DFCS }\end{array}$ \\
\hline Test 1 & 110.3 & 300.01 & 99.99 & 100.01 & 301.80 & 99.56 & 101.12 & -1.10 & -0.41 \\
\hline Test 2 & 110.2 & 99.95 & -100.05 & 100.00 & 102.02 & -101.14 & 101.58 & -1.55 & -0.26 \\
\hline Test 3 & 110.3 & 300.01 & 99.99 & 100.01 & 301.80 & 99.56 & 101.12 & -1.10 & -0.63 \\
\hline Test 4 & 110.3 & 99.95 & -100.05 & 100.00 & 102.02 & -101.14 & 101.58 & -1.55 & -0.46 \\
\hline
\end{tabular}

Table 2: Calibration data of the DFCS

\begin{tabular}{|c|c|c|c|c|c|c|c|c|c|}
\hline $\begin{array}{c}\text { Data of } \\
\text { the } \\
\text { DFCS }\end{array}$ & $\begin{array}{c}\text { Test } \\
\text { frequency } \\
f \\
\\
/ \mathrm{Hz} \\
\end{array}$ & \begin{tabular}{|c}
$\begin{array}{c}\text { Peak } \\
\text { force of } \\
\text { the } \\
\text { machine }\end{array}$ \\
$/ \mathrm{kN}$ \\
\end{tabular} & \begin{tabular}{|c}
$\begin{array}{c}\text { Valley } \\
\text { force of } \\
\text { the } \\
\text { machine }\end{array}$ \\
$/ \mathrm{kN}$ \\
\end{tabular} & $\begin{array}{c}\begin{array}{c}\text { Amplitude } \\
\text { of the } \\
\text { machine } \\
\text { force }\end{array} \\
\text { / kN }\end{array}$ & 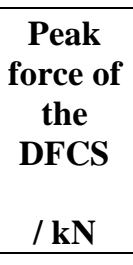 & 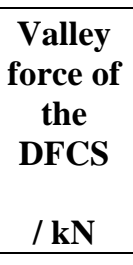 & $\begin{array}{c}\text { Amplitude } \\
\text { of the DFCS } \\
\text { force } \\
\\
/ \mathrm{kN}\end{array}$ & $\begin{array}{c}\begin{array}{c}\text { Error of the } \\
\text { machine }\end{array} \\
\text { amplitude } \\
\text { (uncorrected) } \\
\text { /\% }\end{array}$ & $\begin{array}{c}\text { Error of the } \\
\text { machine } \\
\text { amplitude } \\
\text { (corrected) } \\
/ \%\end{array}$ \\
\hline $\mathrm{Te}$ & & 300.00 & & & & 97.66 & & -2.46 & 1.51 \\
\hline Test & 97.0 & 99.97 & -11 & & 1 & -102 & & & -1.81 \\
\hline Test 3 & & & & & & & & & -1.73 \\
\hline Test 4 & 97.0 & .97 & 100.04 & 100.01 & 102.94 & -102.71 & 102.82 & -2.74 & -2.01 \\
\hline
\end{tabular}

Table 3: Calculation parameters in additional inertial force correction of the DFCS

\begin{tabular}{|c|c|c|c|c|c|c|}
\hline $\begin{array}{l}\text { Calculation parameters of the stiffness } \\
\text { method correction }\end{array}$ & $\begin{array}{l}m_{2} \\
/ \mathbf{k g}\end{array}$ & $\begin{array}{l}\Delta m \\
/ \mathrm{kg}\end{array}$ & $\begin{array}{c}\boldsymbol{k}_{\mathrm{B}} \\
/\left(\mathbf{N} \cdot \mathbf{m}^{-1}\right)\end{array}$ & $\begin{array}{c}\Delta k \\
/\left(\mathbf{N} \cdot \mathbf{m}^{-1}\right)\end{array}$ & $\begin{array}{c}\omega \\
/\left(\operatorname{rad} \cdot \mathbf{s}^{-1}\right)\end{array}$ & $\begin{array}{c}\Delta \omega \\
/\left(\operatorname{rad} \cdot \mathbf{s}^{-1}\right)\end{array}$ \\
\hline Test 1 & 35 & 6 & $3.1 \mathrm{E}+08$ & $-7.0 \mathrm{E}+07$ & 693.0 & -82.3 \\
\hline Test 2 & 35 & 6 & $3.1 \mathrm{E}+08$ & $-7.0 \mathrm{E}+07$ & 692.4 & -82.9 \\
\hline $\begin{array}{l}\text { Calculation parameters of the } \\
\text { acceleration method correction }\end{array}$ & $\begin{array}{l}\mathrm{m}_{2} \\
/ \mathrm{kg}\end{array}$ & $\begin{array}{l}\Delta m \\
/ \mathbf{k g}\end{array}$ & $\begin{array}{c}a \\
/\left(\mathbf{m} \cdot \mathbf{s}^{-2}\right)\end{array}$ & $\begin{array}{c}\Delta a \\
/\left(\mathbf{m} \cdot \mathbf{s}^{-2}\right)\end{array}$ & & \\
\hline Test 3 & 35 & 6 & 43.8 & 12.6 & & \\
\hline Test 4 & 35 & 6 & 43.8 & 12.6 & & \\
\hline
\end{tabular}

\subsection{Discussion}

\section{The Analysis of Data}

According to the test results, the differences between the DFCS results with inertial force corrections and the replica test-piece results are mostly within $0.5 \%$. The differences of individual comparison data exceed $0.5 \%$, which might be caused by the inconsistency between the real measurement position and the ideal measurement position - centre of equivalent inertial mass - in the acceleration measurement. Further studies about the acceleration distribution along the axis of the applied force and the acceleration measurement method are needed.

\section{The Necessity of the DFCS Method}

The calibration method of a replica test-piece has been used according to ISO 4965-1. Replica testpieces are made of one or more test-pieces to be tested, which are bonded with strain gauges. Replica test-pieces are used in dynamic force calibration of fatigue machines after they are calibrated on force standard machines. Because the calibration state is highly consistent with the testing state and there is no additional inertial force caused by external factors, a high accurate calibration result could be 
achieved using the replica test-piece calibration method. But the disadvantages of this method are as follows: (1) different replica test-pieces are needed to be made for different fatigue machines. Different replica test-pieces are also needed to be made for some same specification fatigue machines to be calibrated, only because the materials and specifications of the test-pieces of each fatigue machine are different. Therefore, the production cost of the replica test-pieces is high; (2) because the maximum bearing force of the replica test-piece made of the actual test pieces to be tested on a fatigue machine usually could not cover the full range of the fatigue machine force, the maximum force of the dynamic force range of the fatigue machine could not be calibrated by its own replica test-piece of the fatigue machine; (3) because of the factors of the replica test-piece caused by the strain gauge bonding process, it is difficult to evaluate the uncertainty of the replica test-piece itself.

The advantages of the DFCS calibration method are as follows: (1) the whole dynamic force range of a fatigue machine could be calibrated with a proper DFCS; (2) a DFCS could be reused, and could be used for many different fatigue machines. So it costs low in use and is easy to be promoted; (3) the technology of force sensor is mature, and its own uncertainty could be accurately evaluated for a qualified force sensor; (4) the DFCS method is not only suitable for a typical metallic material fatigue testing machine, but also for some other types of fatigue testing machines and for actuators with single or combined use. The disadvantage of DFCS method is that some additional inertial force influences are induced. In order to overcome this shortcoming, it is necessary to give the inertial force compensation method.

There are two typical types of fatigue machines, which are the resonant fatigue machine and the nonresonant fatigue machine. For each type machine, there are two kinds of configurations, which are Type 1 and Type 2 configuration. So there are mainly 4 kinds of machines widely used so far. The above are the types and structures of the fatigue testing machines. Aiming at the influence of inertial force always existing in fatigue machines, there are two methods of correcting the inertial force, which is the stiffness method and the acceleration method. They are two correction methods which could be selected to use in a calibration.

\section{SUMMARY}

(1) Using a replica test piece that has the same stiffness and mass with the real specimen gives an accurate result of a cyclic force calibration because no other inertial force is induced except the one by the real specimen.

(2) It would be best if the DFCS has exactly the same mass and stiffness as the real specimen, so that no additional inertial force is generated, and no correction is needed.

(3) It is not necessary to strictly limit the mass and stiffness of the DFCS to be consistent with the real specimen. By accurately measuring mass and stiffness or acceleration to correct additional inertial force, the same accurate calibration results could be obtained by using a DFCS different from the real specimen. The difference between the DFCS and the replica test-piece methods is within $\pm 1 \%$.

(4) With an appropriate adaptor, a DFCS could be reused in the calibration for different fatigue machines. Further studies about the acceleration distribution along the axis of the applied force and the acceleration measurement method are needed.

\section{REFERENCES}

[1] ISO 4965-1, "Metallic materials - Dynamic force calibration for uniaxial fatigue testing - Part 1 Testing systems", ISO, Switzerland, 2012.

[2] ASTM E467-08, "Standard Practice for Verification of Constant Amplitude Dynamic Forces in an Axial Fatigue Testing System", ASTM International, USA, 2014.

[3] D. Georgakopoulos, J. M. Williams, A. Knott, T. Esward, P.S. Wright, "Dynamic calibration of fatigue testing machines", in Proc. of British Electromagnetic Measurements Conference, Teddington, UK, 14-17 November 2005. Online [accessed 20201105]: http://eprintspublications.npl.co.uk/3317/1/BEMC 2005-11.pdf 